\title{
Spreading of a surfactant monolayer on a thin liquid film: Onset and evolution of digitated structures
}

\author{
Omar K. Matar ${ }^{\mathrm{a})}$ and Sandra M. Troian ${ }^{\mathrm{b}}$ \\ Department of Chemical Engineering, Princeton University, Princeton, New Jersey 08544
}

(Received 16 July 1998; accepted for publication 30 December 1998)

\begin{abstract}
We describe the response of an insoluble surfactant monolayer spreading on the surface of a thin liquid film to small disturbances in the film thickness and surfactant concentration. The surface shear stress, which derives from variations in surfactant concentration at the air-liquid interface, rapidly drives liquid and surfactant from the source toward the distal region of higher surface tension. A previous linear stability analysis of a quasi-steady state solution describing the spreading of a finite strip of surfactant on a thin Newtonian film has predicted only stable modes. [Dynamics in Small Confining Systems III, Materials Research Society Symposium Proceedings, edited by J. M. Drake, J. Klafter, and E. R. Kopelman (Materials Research Society, Boston, 1996), Vol. 464, p. 237; Phys. Fluids A 9, 3645 (1997); O. K. Matar Ph.D. thesis, Princeton University, Princeton, NJ, 1998]. A perturbation analysis of the transient behavior, however, has revealed the possibility of significant amplification of disturbances in the film thickness within an order one shear time after the onset of flow [Phys. Fluids A 10, 1234 (1998); “Transient response of a surfactant monolayer spreading on a thin liquid film: Mechanism for amplification of disturbances,' submitted to Phys. Fluids]. In this paper we describe the linearized transient behavior and interpret which physical parameters most strongly affect the disturbance amplification ratio. We show how the disturbances localize behind the moving front and how the inclusion of van der Waals forces further enhances their growth and lifetime. We also present numerical solutions to the fully nonlinear 2D governing equations. As time evolves, the nonlinear system sustains disturbances of longer and longer wavelength, consistent with the quasi-steady state and transient linearized descriptions. In addition, for the parameter set investigated, disturbances consisting of several harmonics of a fundamental wavenumber do not couple significantly. The system eventually singles out the smallest wavenumber disturbance in the chosen set. The summary of results to date seems to suggest that the fingering process may be a transient response which nonetheless has a dramatic influence on the spreading process since the digitated structures redirect the flux of liquid and surfactant to produce nonuniform surface coverage. (C) 1999 American Institute of Physics. [S1054-1500(99)02001-7]
\end{abstract}

Surfactant molecules play a vital role in many biological and industrial processes because of their native ability to lower surface tension in proportion to their concentration. Their presence greatly improves the wetting and spreading capability of commonplace substances like inks, paints, and herbicides. Lung surfactants produced in the alveoli are critical in maintaining lung compliance. A deficiency in lung surfactant production, for example, is known to cause pulmonary edema and other respiratory difficulties. When surfactant molecules contact a liquid surface of higher surface tension, a shear stress develops at the air-liquid interface in proportion to the gradient in surfactant concentration. This stress forces liquid and surfactant to flow toward regions of higher surface tension and in the process strongly deforms the underlying liquid film. Experiments by several groups [Chem. Eng. Commun. 13, 133 (1981); Phys. Rev. Lett. 62, 1496 (1989); Langmuir 11, 87 (1995); Phys. Fluids A

\footnotetext{
${ }^{a)}$ Dept. of Chemical Engineering and Chemical Technology, Imperial College, London, England SW7 2BY.

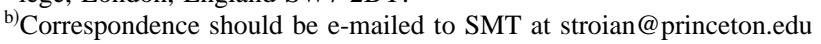

7, 2640 (1995); Faraday Discuss. 104, 307 (1996)] have shown that the spreading of surfactant molecules on athin liquid film often produces unusual digitated structures near the region of initial contact. We briefly review theoretical efforts using linear stability analysis to uncover the source of digitation. We then discuss results from transient growth analyses and direct numerical simulations. The focus of this work rests with the transient spreading behavior - how do small disturbances influence the spreading process at early times? Both the transient and nonlinear studies highlight the importance of regions in the film thickness which thicken (at the monolayer advancing front) or thin (at the surfactant source) in response to the evolving shear stress and whose curvature and higher derivatives becomes substantial. Our studies reveal that the surfactant distribution which develops during the spreading event produces a liquid layer which is highly susceptible to spanwise disturbances. This corrugation is sustained for several shear times but eventually decays away. Although the disturbance speed and mobility is decreased in the thinnest regions of the film, the additional presence of van der Waals forces which promote thinning, re-amplify decay- 


\section{ing disturbances, increase their lifetime, and produce digitated liquid structures.}

\section{INTRODUCTION}

Surfactant molecules placed at an air-liquid interface will spontaneously spread to cover regions of higher surface tension thereby reducing the internal free energy of the liquid system. The surfactant concentration profile and the deformation in film thickness, as well as the speed and uniformity of coverage, are critical factors which affect many coating processes. Empirically, it is well known that surfactant additives can significantly improve wettability and spreading rates. Perhaps less understood until recently is the spreading behavior of a surfactant coated film. Least understood are the unusual fingering patterns which sometimes occur in surfactant films soon after spreading commences. In the absence of disturbances the spreading process can be simply explained. Regions of the air-liquid interface rich in surfactant experience a lower surface tension whose magnitude is proportional to the local concentration. Gradients in the surface concentration at the air-liquid interface give rise to gradients in surface tension which produce a shear stress on the liquid film. This stress forces liquid and surfactant to flow toward regions of higher surface tension, a process described as Marangoni driven flow. ${ }^{1}$ Experimentally and theoretically, it has been shown that surfactants spreading on a thin liquid film (whose lateral extent greatly exceeds its thickness) will drive fluid into a thickened and rapidly advancing ridge with subsequent thinning near the original line of contact between the clean surface and surfactant.

Various experimental studies ${ }^{2-6}$ have shown that once the spreading of surfactant commences, the film thickness near the source region begins to thin and develops a digitated appearance as shown in Fig. 1. The curve describing the perimeter of the pattern shown in Fig. 1(a) has been measured to have a fractal dimension $D_{f} \approx 1.7$, a value which remains constant in time. ${ }^{7}$ The liquid streamers advance over the surface by continually bifurcating at the tips. Although the physical mechanism controlling the spreading process is different than the one responsible for unstable miscible or immiscible viscous fingering, ${ }^{8,9}$ the patterns in both cases appear similar in evolution and shape. For example, the finger-like protrusions shown in Fig. 1 also undergo lateral spreading, shielding, and tip-splitting, features characteristic of viscous fingering patterns. The surfactant patterns are known to occur quite easily and have been found under many different experimental conditions including spreading in rectilinear ${ }^{4,5}$ or axisymmetric geometry, ${ }^{2,3,6}$ spreading from a source held at constant concentration ${ }^{4,5}$ or from a finite reservoir, ${ }^{2,3,6}$ spreading on films ranging in thickness from microns ${ }^{2-6}$ to millimeters, ${ }^{10}$ with soluble $^{2,4,10}$ or insoluble ${ }^{3,5,10}$ surfactants of various head group charge, and with a surfactant source either above $\mathrm{e}^{2,3,10}$ or below ${ }^{3,10}$ the critical micelle concentration. This latter concentration defines the point at which the air-liquid interface can no longer accommodate surfactant thereby forcing excess surface molecules into the bulk liquid to form micellar clusters.

During the past several years a lubrication model has

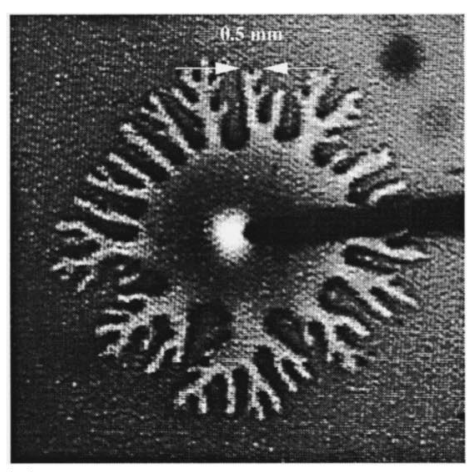

(a)

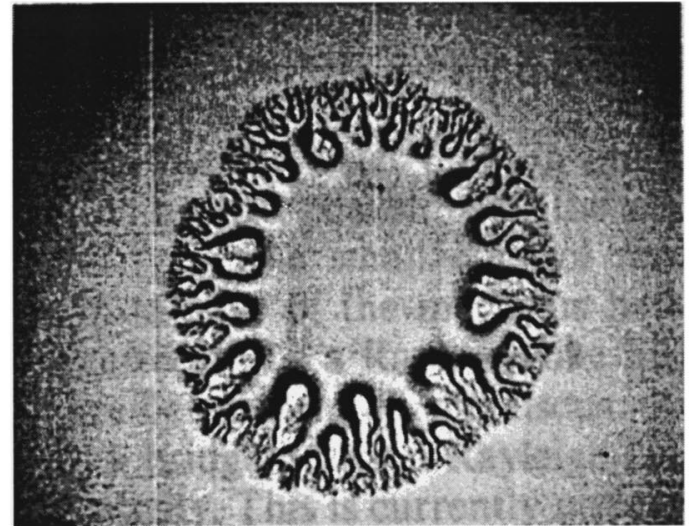

(b)

FIG. 1. Surface patterns produced during the spreading of a surfactant drop on a thin water film. (a) $2 \mu \mathrm{l}$ drop of $1 \mathrm{mM}$ aqueous AOT surfactant spreading on a film approximately $1 \mu \mathrm{m}$ in thickness (Ref. 7). Diameter of the fingering pattern is approximately $0.9 \mathrm{~cm}$ and corresponds to a time $0.2 \mathrm{sec}$ after deposition. (b) Drop of $C_{12} E_{10}$ surfactant in ethylene glycol spreading on a film approximately $10^{-2} \mu$ in thickness (Ref. 6). The advancing liquid ridge normally located ahead of the fingering patterns is not visible in these photographs.

been developed to describe the spreading behavior of a surfactant film on a thin liquid layer. The surfactant can be delivered either as a hemispherical cap at constant concentration ${ }^{11}$ or as a monolayer ${ }^{12-17}$ emanating from a finite or infinite reservoir. This original model has been extended to include not only Marangoni stresses and capillarity but also gravity, surface diffusion, bulk diffusion, van der Waals forces, and the presence of endogenous (pre-existing) surfactant on the initial liquid film. The base state solutions (solutions in the absence of disturbances) governing the behavior of the film thickness during the spreading process predict a propagating thickened ridge at the surfactant front and significant thinning near the source region. The exact shape of the thickness and concentration profiles depend, of course, on the characteristic dimensionless numbers describing the relative influence of the various forces affecting the spreading.

The first effort at understanding the development of the liquid digitation derived impetus from the similarity between the surfactant patterns and viscous fingering. Within an approximation that only allowed disturbances in the surfactant concentration (and not the film thickness), it was shown that the disturbance equation governing the flow near the base of 
a surfactant droplet was dominated by a term proportional to the Laplacian of the concentration field. Drawing analogy with the linear stability analysis of viscous fingering, the surfactant boundary at the base of the droplet was shown to be linearly unstable to transverse disturbances of small wavenumber, $K$, with capillary corrections setting an upper bound on $K .^{11}$ This simplified model identified Marangoni stresses, arising from the distribution of insoluble surfactant at the interface, as the source of instability. The role played by the Laplacian of the surfactant concentration was likened to the destabilizing role played by the Laplacian of the external pressure in viscous fingering.

Since the surfactant patterns are known to occur in many different geometries, Matar and Troian ${ }^{18,19}$ later considered a simple geometry consisting of a finite, rectilinear strip of insoluble surfactant spreading on a thin liquid film. Initially all other forces aside from Marangoni stresses were ignored. In this limit, the base flow solutions for the film thickness and surfactant concentration assume a self-similar form in a stretched coordinate system whose range is coincident with the leading edge of the monolayer. Since the rate of change of the base state scales as $t^{-4 / 3}$, it was therefore assumed that at sufficiently long times, the base states could be regarded as quasi-steady. Within this approximation, the film thickness and surfactant concentration were found to be linearly stable to disturbances of all wavenumbers. This prediction, which is only valid as $t \rightarrow \infty$, seemed to belie experimental observations showing spanwise structuration of an initially flat and uniform liquid layer. The linear stability analysis was later modified to include capillarity and surface diffusion, ${ }^{20}$ two forces that produce more realistic base state profiles for the film thickness and surfactant concentration. These profiles were advanced in time until they too approached self-similar form. Once again, however, the largest eigenvalue describing the disturbance growth rate, was negative in most cases. There did appear a very small (of order $10^{-3}$ or less) and positive eigenvalue for the $K=0$ mode only, but for very long spreading times or for relevant values of the capillary and Peclet numbers, this eigenvalue became negative. ${ }^{20}$

These earlier computations focused primarily on the dynamics of the spreading process at very late times. An understanding of the early time dynamics, however, is perhaps even more critical since the magnitude of the surfactant concentration gradient, and hence the driving force for the spreading event, is largest at early times. Unfortunately, the large initial stress the system experiences produces base states which develop a spatially complex shape during this initial period. Because the linearized operators, $\mathcal{L}$, governing the evolution of disturbances contain terms which depend on the spatially inhomogeneous base states and their derivatives, the product, $\mathcal{L} \mathcal{L}^{\dagger}$, is noncommutative. The behavior of this system at early times cannot therefore be predicted by classical eigenanalysis since the operators governing the disturbances are non-normal. ${ }^{19-22}$

As explained previously by several authors ${ }^{23,24}$ and known since the early part of this century, ${ }^{25}$ the eigenvalues corresponding to non-normal operators strictly describe the asymptotic behavior of a system as $t \rightarrow \infty$. Infinitesimal dis-

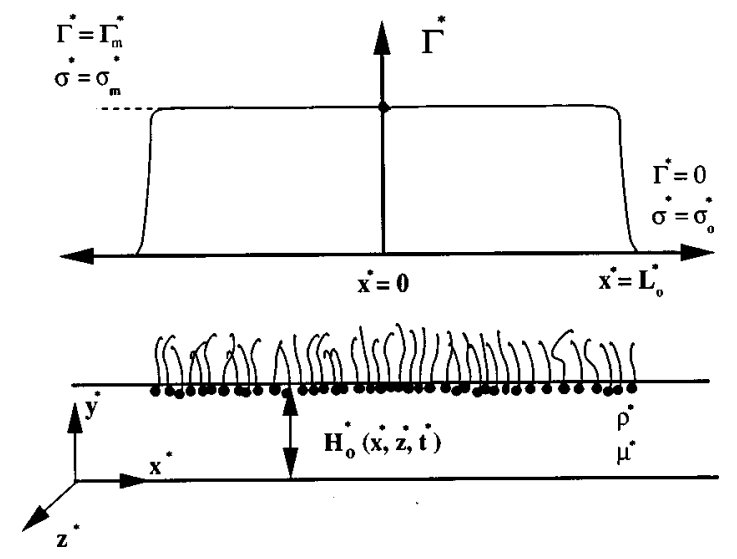

FIG. 2. Schematic diagram of the monolayer deposition process.

turbances may undergo considerable growth at early or intermediate times even in systems achieving eventual asymptotic decay. Indeed, as we describe below, a spreading surfactant coated film can undergo large disturbance amplification on the order of a few Marangoni shear times. We review below the transient behavior of the linearized system of equations governing the dynamics of the film thickness and surfactant concentration and discuss the effect of varying different physical parameters of the flow. In particular, the inclusion of van der Waals forces accelerates the thinning in both the streamwise and transverse flow directions. Once the film has sufficiently thinned, the amplification of disturbances, which was previously found to decay in time, rises sharply at intermediate times. We have also integrated the fully nonlinear equations to explore the possibility of mode coupling and the development of disturbances not captured by the linearized description. For the parameter values and initial conditions used, these results simply reconfirm the picture obtained within the linearized model.

\section{GOVERNING EQUATIONS}

We consider an incompressible, Newtonian film of uniform thickness $H^{*}\left(x^{*}, z^{*}, t^{*}=0\right)=H_{0}^{*}$, viscosity $\mu^{*}$, and density $\rho^{*}$ supported by a flat, rigid substrate at $y^{*}=0$, as shown in Fig. 2. The liquid surface is initially only partially covered by an insoluble surfactant monolayer whose surface concentration, $\Gamma^{*}\left(x^{*}, z^{*}, t^{*}\right)$, achieves its maximum value, $\Gamma_{m}^{*}$, at the origin $x^{*}=0$ vanishing smoothly at $x^{*}=L_{0}^{*}$. The smallest value in surface tension corresponding to $\Gamma_{m}^{*}$ is denoted by $\sigma_{m}^{*}$ while the largest value, $\sigma_{0}^{*}$, denotes the surface tension of the uncontaminated liquid surface. The equations of motion are derived in the lubrication approximation for which $\varepsilon=H_{0}^{*} / L_{0}^{*} \ll 1$. The monolayer and underlying fluid spread spontaneously toward the uncontaminated region under the action of an initial gradient in surface tension characterized by $\Pi^{*} / L_{0}^{*}=\left(\sigma_{0}^{*}-\sigma_{m}^{*}\right) / L_{0}^{*}$, where $\Pi^{*}$ is the spreading pressure. This flow is counter-balanced by the viscous stress at the surface which is of order $\mu^{*} U^{*} / H_{0}^{*}$. The spreading velocity characteristic of Marangoni driven flow in thin films is determined from this tangential stress balance to be $U^{*}=\varepsilon \Pi * / \mu^{*}$. 
The equations are reduced to dimensionless form by introducing characteristic scales relevant to the dynamics of a thin liquid film driven to spread by Marangoni stresses. The horizontal coordinates, $x^{*}$ and $z^{*}$, are scaled by the length of the initial surfactant strip, $L_{0}^{*}$; the vertical coordinate, $y^{*}$, is scaled by the initial film thickness, $H_{0}^{*}$. Within the lubrication approximation, the axial and transverse velocities, $u^{*}$ and $w^{*}$, are scaled by $U^{*}$ while the vertical velocity, $v^{*}$, is scaled by $\varepsilon U^{*}$. The characteristic time scale is given by $L_{0}^{*} / U^{*}$ and the characteristic pressure acting throughout the film is given by $\mu^{*} U^{*} L_{0}^{*} / H_{0}^{* 2}=\Pi * / H_{0}^{*}$. The local surface concentration and surface tension are normalized by their values at the origin, namely $\Gamma_{m}^{*}$ and $\sigma_{m}^{*}$. Likewise, the local driving force for spreading is normalized by $\Pi^{*}$.

The inertial terms in the Navier Stokes equation can be ignored provided $\varepsilon^{2} \operatorname{Re} \ll 1$. In this system, both $\varepsilon$ and the Reynolds number, $\operatorname{Re} \equiv\left(\rho^{*} U^{*} H_{0}^{*}\right) / \mu^{*}=\left(\rho^{*} \Pi^{*} \varepsilon^{2} L_{0}^{*}\right) /$ $\mu^{* 2}$, are vanishingly small. For sufficiently thin films, the Bond number, $\mathrm{Bo} \equiv \rho^{*} g^{*} H_{0}^{* 2} / \Pi^{*}$, is negligible. There are three relevant dimensionless numbers appearing in the equations of motion which reflect the importance of surface curvature, surface diffusion (represented by the diffusion coefficient $\mathcal{D}_{s}^{*}$ ), and van der Waals forces. These numbers are defined by an inverse modified capillary number, $\mathcal{C}$ $\equiv \varepsilon^{2} \sigma_{m}^{*} / \Pi^{*}$ (related to the usual capillary number, $\mathrm{Ca}$ $\equiv \mu^{*} U^{*} / \sigma_{m}^{*}$, by $\mathcal{C}=\varepsilon^{3} / \mathrm{Ca}$ ), a surface Peclet number, $\mathrm{Pe}_{s}$ $\equiv U^{*} L_{0}^{*} / \mathcal{D}_{s}^{*}=\Pi^{*} H_{0}^{*} / \mu^{*} \mathcal{D}_{s}^{*}$, and a modified Hamaker constant, $\mathcal{A} \equiv \widetilde{A} / 6 \pi \Pi^{*} H_{0}^{* 2}$. The two coupled equations describing the spatio-temporal evolution of the film thickness and surfactant concentration are defined by Eqs. (1) and (2) below. Complete details of the derivation leading to these differential equations can be found elsewhere. ${ }^{13,14,18-20,22}$

$$
\begin{aligned}
H_{t} & +\frac{1}{2} \nabla \cdot\left(H^{2} \nabla \sigma\right)+\frac{\mathcal{C}}{3} \nabla \cdot\left(H^{3} \nabla^{3} H\right) \\
& +\mathcal{A} \boldsymbol{\nabla} \cdot\left(\frac{1}{H} \nabla H\right)=0, \\
\Gamma_{t}+ & \nabla \cdot(H \Gamma \nabla \sigma)+\frac{\mathcal{C}}{2} \nabla \cdot\left(\Gamma H^{2} \nabla^{3} H\right) \\
& +\frac{3}{2} \mathcal{A} \boldsymbol{\nabla} \cdot\left(\frac{\Gamma}{H^{2}} \nabla H\right)=\frac{1}{\mathrm{Pe}_{s}} \nabla^{2} \Gamma .
\end{aligned}
$$

Although $\mathcal{C}$ scales with $\varepsilon^{2}$, the capillary terms are retained since the surface curvature achieves values of order $O\left(\varepsilon^{-2}\right) \cdot{ }^{26}$ Equations (1) and (2) are coupled by the surfactant equation of state which relates the surface tension to the local surfactant concentration. Since the monolayer rapidly expands to cover the entire liquid surface, we choose the simplest relation appropriate for molecules in a "gaseous state," namely $\sigma=1-\Gamma$.

\section{MATHEMATICAL DESCRIPTION OF SPREADING DYNAMICS}

The response of the system to small disturbances is obtained by linearizing Eqs. (1) and (2) according to

$$
H(x, z, t)=H_{0}(x, t)+\widetilde{H}(x, z, t),
$$

$$
\Gamma(x, z, t)=\Gamma_{0}(x, t)+\widetilde{\Gamma}(x, z, t),
$$

in which $\widetilde{H}$ and $\widetilde{\Gamma}$ represent infinitesimal disturbances in the film thickness and surfactant concentration, respectively.

\section{A. Linearized formulation}

\section{Base states}

In the absence of disturbances $(\widetilde{H}=\widetilde{\Gamma}=0)$, the spreading is simply a function of $x$ and $t$ and Eqs. (1) and (2) reduce to the form

$$
\begin{aligned}
H_{0_{t}}= & \frac{1}{2}\left(H_{0}^{2} \Gamma_{0_{x}}\right)_{x}-\frac{\mathcal{C}}{3}\left(H_{0}^{3} H_{0_{x x x}}\right)_{x}-\mathcal{A}\left(\frac{1}{H_{0}} H_{0_{x}}\right)_{x}, \\
\Gamma_{0_{t}}= & \left(\Gamma_{0} H_{0} \Gamma_{0_{x}}\right)_{x}+\frac{1}{\mathrm{Pe}_{s}} \Gamma_{0_{x x}}-\frac{\mathcal{C}}{2}\left(\Gamma_{0} H_{0}^{2} H_{0_{x x x}}\right)_{x} \\
& -\frac{3}{2} \mathcal{A}\left(\frac{\Gamma_{0}}{H_{0}^{2}} H_{0_{x}}\right)_{x} .
\end{aligned}
$$

The subscript " 0 "' will henceforth refer to the base flow solutions (undisturbed states). Other subscripts will refer to partial differentiation in space or time. The coupled equations (5) and (6) are solved subject to the following boundary conditions at the origin, $x=0$, and far downstream, $x \rightarrow \infty$ :

$$
\begin{aligned}
& H_{0 x}(0, t)=0, \quad H_{0 x x x}(0, t)=0, \quad \text { and } \Gamma_{0 x}(0, t)=0, \\
& H_{0}(\infty, t)=1, \quad H_{0 x}(\infty, t)=0, \quad \text { and } \Gamma_{0}(\infty, t)=0 .
\end{aligned}
$$

The constraints at the origin reflect symmetry and no-flux about $x=0$ while the far field constraints reflect undisturbed conditions far from the advancing surfactant front. The base state initial conditions, chosen to mimic a flat, uniform liquid film partially covered by a surfactant monolayer of constant concentration, $A_{L}$, are given by

$$
H_{0}\left(x, t_{0}\right)=1, \quad \Gamma_{0}\left(x, t_{0}\right)=0.5 A_{L}\left[1-\tanh \left(B_{L}\left(x-x_{0}\right)\right)\right] .
$$

The surfactant concentration resembles a top hat distribution which vanishes smoothly near $x=x_{0}$. Although this choice of initial and boundary conditions (the far field constraints in particular) represent the desired experimental conditions, they preclude the possibility of steady-state base flow solutions.

\section{Disturbance equations}

We seek disturbances of the form $(\widetilde{H}, \widetilde{\Gamma})(x, z, t)$ $=(\Psi, \Phi)(x, t) e^{i K z}$, describing a function which propagates and evolves in time in the streamwise direction while exhibiting sinusoidal character in the transverse direction. The disturbances $\Psi$ and $\Phi$ are determined from the following set of equations representing a disturbance of transverse wavenumber $K$ : 


$$
\begin{aligned}
\Psi_{t}=\mathcal{L}_{1}[\Psi, \Phi]= & \frac{1}{2}\left(H_{0}^{2} \Phi_{x}+2 H_{0} \Gamma_{0 x} \Psi\right)_{x}-\frac{K^{2}}{2} H_{0}^{2} \Phi-\frac{\mathcal{C}}{3}\left[\left(H_{0}^{3} \Psi_{x x x}+3 H_{0}^{2} H_{0_{x x x}} \Psi\right)_{x}-K^{2}\left(\left(H_{0}^{3}\right)_{x} \Psi_{x}+2 H_{0}^{3} \Psi_{x x}\right)+K^{4} H_{0}^{3} \Psi\right] \\
& -\mathcal{A}\left[\left(2\left(H_{0_{x}}\right)^{2}-H_{0} H_{0_{x x}}-K^{2} H_{0}^{2}\right) \Psi-2 H_{0} H_{0_{x}} \Psi_{x}+H_{0}^{2} \Psi_{x x}\right] / H_{0}^{3}, \\
\Phi_{t}=\mathcal{L}_{2}[\Psi, \Phi]= & \left(\Gamma_{0} \Gamma_{0 x} \Psi+H_{0} \Gamma_{0 x} \Phi+\Gamma_{0} H_{0} \Phi_{x}\right)_{x}-K^{2} \Gamma_{0} H_{0} \Phi+\frac{1}{\mathrm{Pe}_{s}}\left(\Phi_{x x}-K^{2} \Phi\right) \\
& -\frac{\mathcal{C}}{2}\left(\Gamma_{0} H_{0}^{2} \Psi_{x x x}+2 \Gamma_{0} H_{0} H_{0_{x x x}} \Psi+H_{0}^{2} H_{0_{x x x}} \Phi\right)_{x}-\frac{\mathcal{C}}{2}\left(-K^{2}\left(\left(\Gamma_{0} H_{0}^{2}\right)_{x} \Psi_{x}+2 \Gamma_{0} H_{0}^{2} \Psi_{x x}\right)+K^{4} \Gamma_{0} H_{0}^{2} \Psi\right) \\
& -\frac{3}{2} \mathcal{A}\left[\left(6 \Gamma_{0}\left(H_{0_{x}}\right)^{2}-2 H_{0} H_{0_{x}} \Gamma_{0_{x}}-2 H_{0} \Gamma_{0} H_{0_{x x}}-K^{2} \Gamma_{0} H_{0}^{2}\right) \Psi\right] / H_{0}^{4} \\
& -\frac{3}{2} \mathcal{A}\left[\left(-4 H_{0} \Gamma_{0} H_{0_{x}}+H_{0}^{2} \Gamma_{0_{x}}\right) \Psi_{x}+\Gamma H_{0}^{2} \Psi_{x x}\right] / H_{0}^{4}-\frac{3}{2} \mathcal{A}\left[\left(-2 H_{0} H_{0_{x}}^{2}+H_{0}^{2} H_{0_{x x}}\right) \Phi+H_{0}^{2} H_{0_{x}} \Phi_{x}\right] / H_{0}^{4} .
\end{aligned}
$$

The linearized operators, $\mathcal{L}_{1}$ and $\mathcal{L}_{2}$, are nonautonomous since they depend on the temporal behavior of the base states. For reasons similar to the choice of base state conditions, the boundary conditions applied to the disturbance functions satisfy

$$
\begin{aligned}
& \Psi_{x}(0, t)=0, \quad \Psi_{x x x}(0, t)=0, \quad \text { and } \Phi_{x}(0, t)=0, \\
& \Psi(\infty, t)=0, \quad \Psi_{x}(\infty, t)=0, \quad \text { and } \Phi(\infty, t)=0 .
\end{aligned}
$$

The initial conditions for $\Psi$ and $\Phi$ are given by

$$
\Psi\left(x, t_{0}\right)=\Phi\left(x, t_{0}\right)=e^{-C_{L}\left(x-x_{s}\right)^{2}} .
$$

The disturbances in the film thickness and surfactant concentration represent Gaussian distributions centered at $x=x_{s}$. In this paper, we limit our studies to disturbances applied ahead of the initial monolayer such that $x_{s}>x_{0}$. Since the disturbance equations are linear, the amplitudes of $\Psi$ and $\Phi$ can be set to unity with no loss in generality. Other choices of initial conditions have produced the same qualitative behavior described below. ${ }^{20-22}$

\section{Quantifiers of transient amplification}

As described in the Introduction, the inherent time dependence of the base state governed by Eqs. (5) and (6) precludes a straightforward modal analysis. In addition, the prediction of asymptotic stability for the quasi-steady-state solutions ${ }^{18-20}$ does not rule out the possibility of large transient growth at early or intermediate time scales. In considering the "stability" of a time variant base state, however, one must consider the evolution of a disturbance by comparison with the evolution of the base state itself. For example, if a disturbance decreases in time but the base state decreases at a faster rate, then the disturbance will appear amplified at later times. Conversely, if a disturbance increases in time but the base state increases faster still, then the disturbance will appear to decay in time. ${ }^{27}$ Since the tendency toward stability or instability at any instant in time is all that can be reliably stated in the case of unsteady base states, Shen ${ }^{27}$ proposed the concept of "momentary stability" and an associated growth rate criterion in order to quantify the instantaneous stability of time variant base flows.
We introduce the following quantifiers of transient growth or amplification. One useful measure of the mechanical energy contained in the base flow or an applied disturbance is

$$
E_{q} \equiv \frac{1}{2} \int_{0}^{\infty} q^{2}(x, t) d x, \text { where } q=\Psi, \Phi, H_{0}, \Gamma_{0} .
$$

The ratio of the disturbance energy to that contained in the evolving base state is described by

$$
M_{i}(t) \equiv \frac{E_{i}(t)}{E_{j}(t)}, \quad \text { where }(i, j)=\left(\Psi, H_{0}\right) \text { or }\left(\Phi, \Gamma_{0}\right) .
$$

The disturbance amplification, defined as the energy ratio at time $t$ compared to its initial value at $t_{0}$, is given by

$$
G_{i}(t) \equiv \frac{M_{i}(t)}{M_{i}\left(t_{0}\right)}, \quad \text { where }(i, j)=\left(\Psi, H_{0}\right) \text { or }\left(\Phi, \Gamma_{0}\right) .
$$

Along with these definitions, the associated time-dependent "growth rate" is represented by

$$
\Omega_{i} \equiv \frac{1}{G_{i}} \frac{d G_{i}}{d t}, \text { where } i=\Psi \text { or } \Phi .
$$

Equation (18) is used as a criterion of "momentary stability" in the sense of Shen, ${ }^{27} \Omega_{i}>0$ describes momentary instability while $\Omega_{i}<0$ describes momentary stability. The criterion for asymptotic stability, determined from eigenvalue analysis of the non-normal operators, would correspond to $\lim _{t \rightarrow \infty} \Omega_{i} \leqslant 0 .^{21,22,27}$

\section{Solution procedure}

Equations (5), (6), (10), and (11) are solved simultaneously by the method of lines ${ }^{28}$ for given values of $\mathcal{C}, \mathrm{Pe}_{s}$, $\mathcal{A}$, and $K$. The spatial derivatives were approximated by second-order central finite differences. The equations were updated in time using Gear's method implemented through the ODE solver LSODE. ${ }^{29}$ The number of grid points used in the computations varied between 201 and 501 depending on 


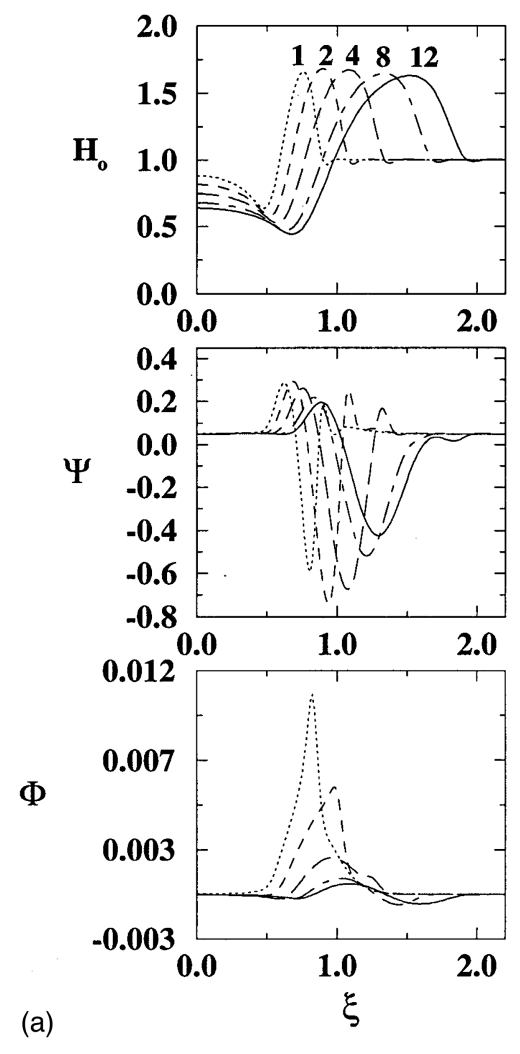

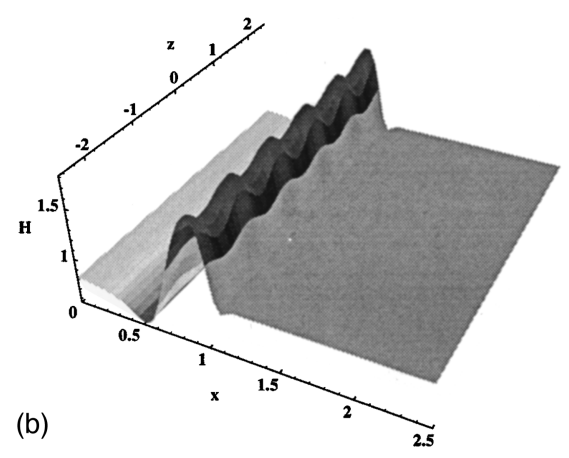

(c)

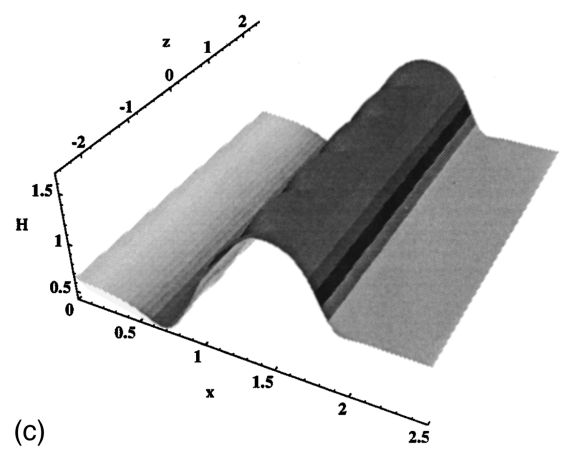

FIG. 3. (a) Base state solution, $H_{0}(x, t)$, with evolved disturbances, $\Psi(x, t)$ and $\Phi(x, t)$, for $K=5, \mathrm{Pe}_{s}$ $=500, \mathcal{C}=10^{-5}$, and $\mathcal{A}=0$. The time $\Delta t=1,2,4,8$, and 12 refers to time after surfactant deposition. (b) and (c) 3-D re-constructions of the total film thickness [with an applied disturbance of $K=5$ for $\Delta t=2$ (b) and $\Delta t=12$ (c)]. Additional parameter values are: $t_{0}=1, A_{L}=0.1, B_{L}=30, C_{L}=12, x_{0}$ $=0.4, x_{s}=0.7$, and $N=301$. the degree of resolution required to produce the full structure of the base states. Solution convergence was easily obtained by refining the spatial grid.

\section{B. Nonlinear formulation}

The fully nonlinear Eqs. (1) and (2) were also integrated directly to yield the spatio-temporal evolution of specified states in the film thickness and surfactant concentration described by

$$
\begin{aligned}
& H\left(x, z, t_{0}\right)=H_{0}\left(x, t_{0}\right)+\delta H\left(x, z, t_{0}\right), \\
& \Gamma\left(x, z, t_{0}\right)=\Gamma_{0}\left(x, t_{0}\right)+\delta \Gamma\left(x, z, t_{0}\right) .
\end{aligned}
$$

The functions $H_{0}\left(x, t_{0}\right)$ and $\Gamma_{0}\left(x, t_{0}\right)$ define the film thickness and concentration profiles at some specified time $t$ $=t_{0}$. For $t_{0}=1$ (the origin of time), these functions are simply given by Eq. (9). For later times $t_{0}>1, H_{0}\left(x, t_{0}\right)$ and $\Gamma_{0}\left(x, t_{0}\right)$ represent the numerical solutions to Eqs. (5) and (6) evolved through $t_{0}$. The disturbances, $\delta H$ and $\delta \Gamma$, were chosen to have a generic form

$$
\begin{aligned}
& \delta H\left(x, z, t_{0}\right)=\widehat{\delta H} e^{-C_{N}\left(x-x_{s}\right)^{2}} R(z), \\
& \delta \Gamma\left(x, z, t_{0}\right)=\widehat{\delta \Gamma} e^{-C_{N}\left(x-x_{s}\right)^{2}} R(z),
\end{aligned}
$$

where the subscript " $N$ ' refers to the parameter value used in the nonlinear computations. The function $R(z)$ represents a summation of sinusoidal functions according to $R(z)$ $=\sum_{j=1}^{j=m} \cos \left(2 \pi K_{j}(z / W)\right)$, where $K_{j}$ is the wavenumber of an applied disturbance and $W$ is the width of the computational domain. The choice of cosines assures that the functions given by Eq. (19) satisfy no flux conditions at $z=0$ and $z$ $=W$. The summation of sinusoidal waves is useful for prob- ing the degree of mode coupling this dynamical system might allow. To make a direct comparison with the results of the linearized theory, the amplitudes of the increments, $\widehat{\delta H}$ and $\widehat{\delta \Gamma}$, are given by

$$
\begin{aligned}
& \widehat{\delta H}=\varepsilon\left[\max _{x} H_{0}\left(x, t_{0}\right)\right], \\
& \widehat{\delta \Gamma}=\varepsilon\left[\max _{x} \Gamma_{0}\left(x, t_{0}\right)\right] .
\end{aligned}
$$

The magnitude of $\varepsilon$ (not to be confused with the lubrication parameter described in Sec. II) in the computations ranged from 0.01 to 0.03 . Disturbances of larger initial amplitude, $\varepsilon$, gave rise to numerical instabilities and were not pursued further. The same physical boundary conditions described in Sec. III A were used to solve Eqs. (1) and (2). In addition, periodicity in the transverse direction was enforced by $H(x, 0, t)=H(x, W, t)$ and $\Gamma(x, 0, t)=\Gamma(x, W, t)$.

We investigated various combinations of the parameter list given by $A_{L}, B_{L}, C_{L}, C_{N}, x_{0}, x_{s}, K$, and $t_{0}$ for experimentally relevant values of the dimensionless numbers $\mathcal{C}$, $\mathrm{Pe}_{s}$, and $\mathcal{A}$. The method of lines was used to integrate the equations of motion and the resultant ODE's were solved using LSODE. The Gear integrator option, which is a variablestep, variable-order, implicit method known to handle stiff ODE's reliably, was originally implemented. This choice lead to computations of order $O\left(N_{x}^{2} N_{z}^{2}\right)$, where $N_{x}$ and $N_{z}$ represent the number of grid points in the $x$ and $z$ directions. When the number of grid points necessary to resolve particularly steep fronts at the advancing monolayer (particularly for large $\mathrm{Pe}_{s}$ and small $\mathcal{C}$ ) were as large as $N_{x}=121$ and $N_{z}=241$, we switched to the Adams option, an explicit 

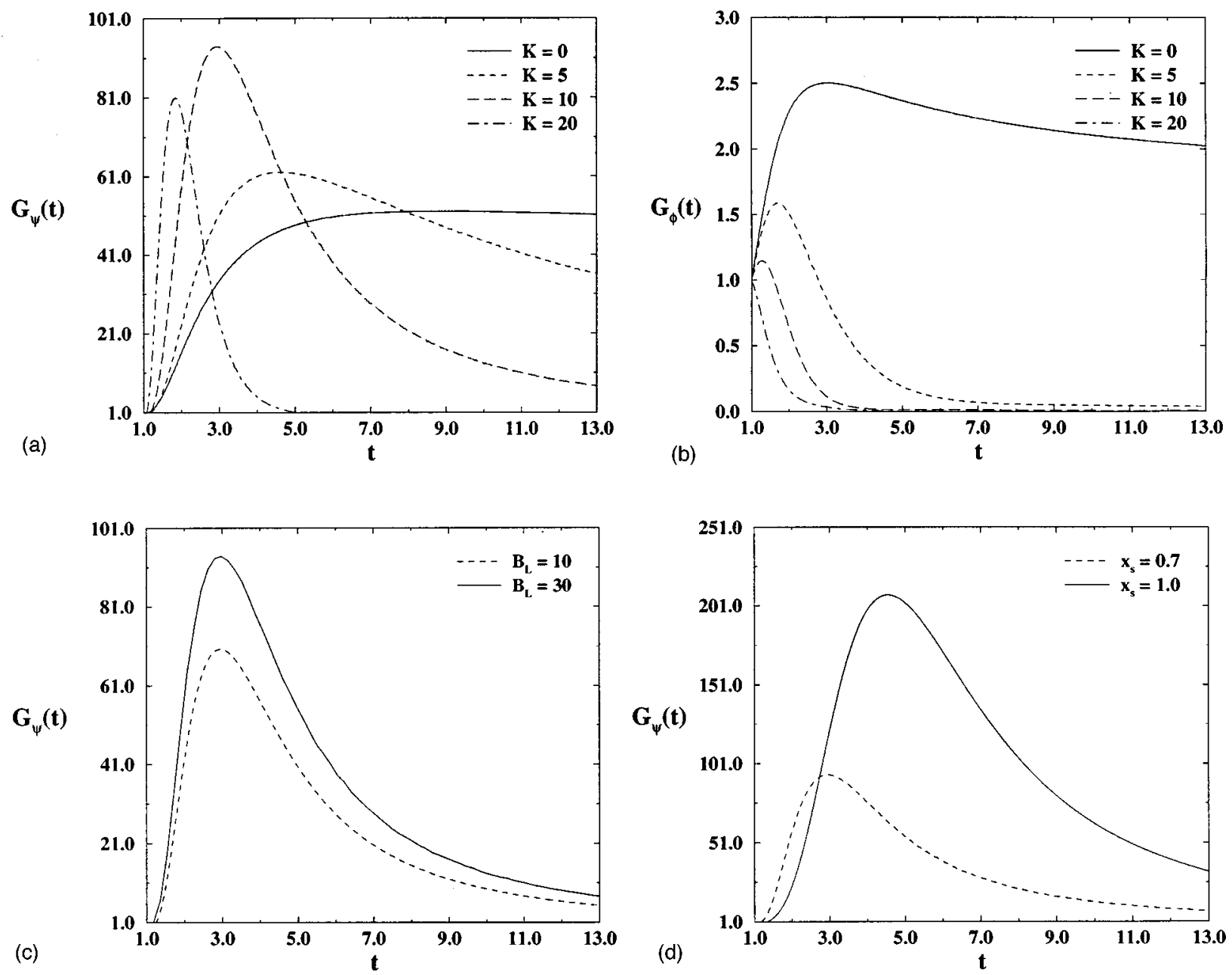

FIG. 4. Amplification ratios, $G_{\Psi}$ and $G_{\Phi}$, corresponding to the linearized transient growth for $\mathrm{Pe}_{s}=500, \mathcal{C}=10^{-5}$, and $\mathcal{A}=0$. (a) and (b) The behavior of $G_{\Psi}$ and $G_{\Phi}$ for various wavenumber disturbances. (c) The dependence of $G_{\Psi}$ on the parameter $B_{L}$, which controls the initial shear stress. (d) The dependence of $G_{\Psi}$ on the parameter $x_{s}$, which defines the central location of the applied disturbance. The remaining parameter values are given in Fig. 3 .

scheme appropriate for stiff equations provided the step sizes are very small. With this choice, the computations reduce to order $O\left(N_{x} N_{z}\right)$. The large number of grid points place an effective restriction on the magnitude of $\mathrm{Pe}_{s}$ and $\mathcal{C}$, which in these studies spanned the range $\mathrm{Pe}_{s} \leqslant 5000$ and $\mathcal{C} \leqslant 10^{-5}$. The grid sizes used in the computations ranged from 0.01 to 0.02 ; solution convergence was monitored by grid size refinement. The computational runs required anywhere from a few minutes to a few hours on an SGI INDY (R4400SC), depending on the parameter values used. As a performance check of this algorithm, we explicitly verified that for the same parameter choices, both the 2-D direct integration of Eqs. (1) and (2) obtained with the explicit Adams method and the 1-D solution to Eqs. (5) and (6) using the implicit Gear's method produced the same results. ${ }^{20}$

\section{RESULTS AND DISCUSSION}

\section{A. Linearized transient response}

We first consider the general character of the solutions to the linearized disturbance equations. Shown in Fig. 3(a) are several snapshots of the base state, $H_{0}$, and the disturbance functions, $\Psi$ and $\Phi$, corresponding to times $\Delta t=1,2,4,8$ and 12 after spreading commences for parameter values $\mathcal{C}$ $=10^{-5}, \mathrm{Pe}_{s}=500$, and $\mathcal{A}=0$. As time evolves, the solutions broaden and decay since the driving force for spreading, which is proportional to the gradient in concentration, continually diminishes. Figures 3(b) and 3(c) represent 3-D visualizations of the sum $H_{0}(x, t)+0.01 \Psi(x, t) \cos (K z)$ at $\Delta t$ $=2$ and 12 (integration commenced at $t_{0}=1$ ) for $K=5$. The disturbance in film thickness, first situated ahead of the surfactant monolayer, is intensified by the passage of the advancing wavefront but eventually decays and localizes behind the steep and rapidly moving front. As observed in experiment, the film corrugations develop a transverse digitation which localizes behind the advancing ridge. Figures 4(a) and 4(b) document the transient growth and decay experienced by the spreading film and surfactant concentration for the same parameter values but various wavenumber disturbances. Large transient growth in $G_{\Psi}(t)$ is evident on the order of a few shear times. The large wavenumber disturbances dominate the response in $G_{\Psi}(t)$ at early times both in amplitude and growth rate (not shown) $)^{20,22}$ but eventually fade away. The smaller wavelength disturbances grow more slowly and achieve smaller amplitudes although they persist for longer times. In contrast to these observations, Fig. 4(b) 

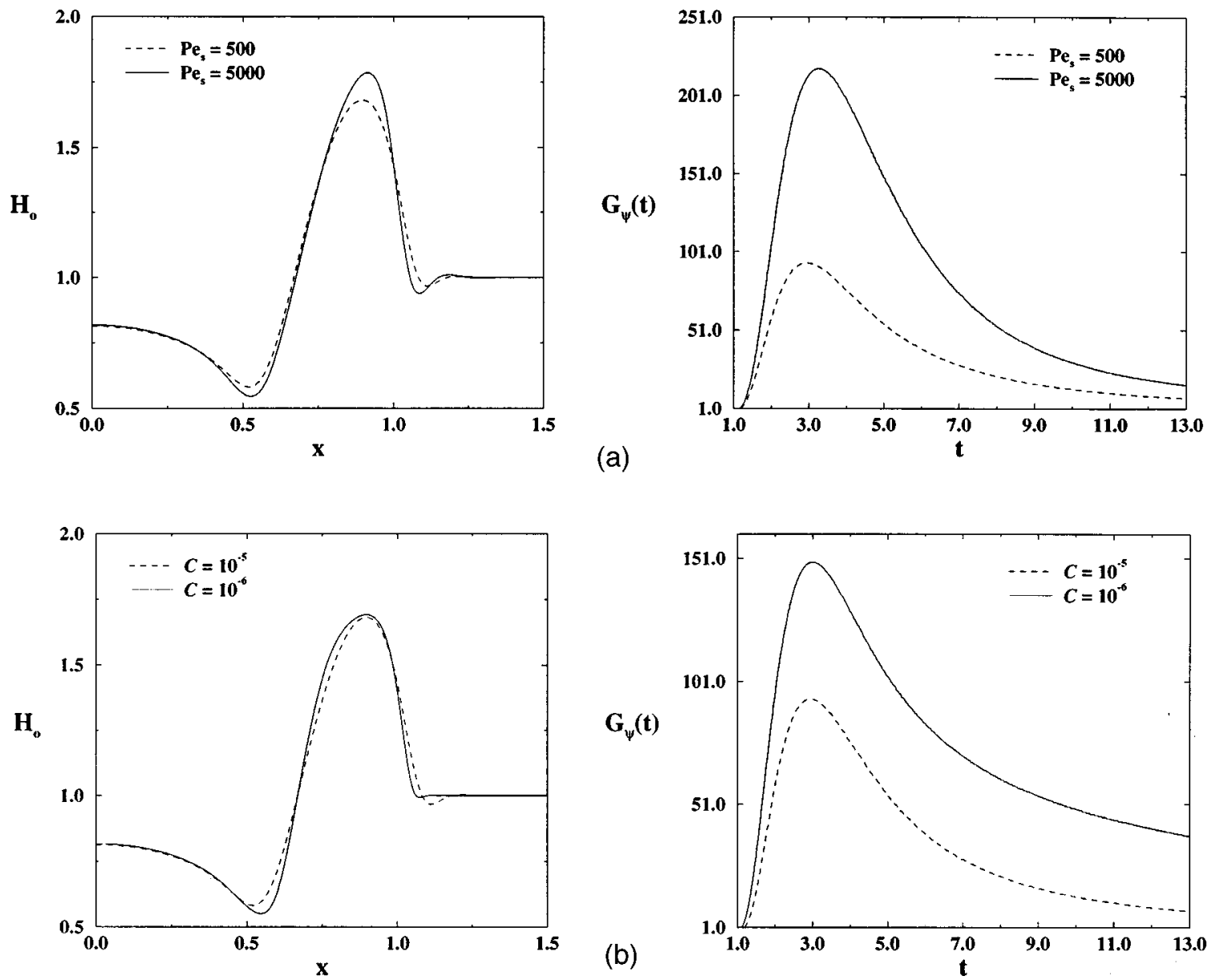

FIG. 5. Linearized response and transient growth of an applied disturbance with $K=10$. (a) The effect of increasing $\mathrm{Pe}_{s}$ on the base state, $H_{0}$ (at $t=3$ ), and on the corresponding amplification ratio, $G_{\Psi}(t)$. The capillary constant is held fixed at $\mathcal{C}=10^{-5}$. (b) The effect of decreasing $\mathcal{C}$ on the base state, $H_{0}$ (at $t$ $=3$ ), and on the corresponding amplification ratio, $G_{\Psi}(t)$. The surface Peclet number is held constant at $\mathrm{Pe}_{s}=500$. The remaining parameter values are given in Fig. 3.

indicates that $G_{\Phi}$ does not experience any significant enhancement. This behavior can be understood by appealing to the nature of the surface shear stress caused by concentration gradients. Even a very small redistribution of surfactant on the surface can have a large effect on the film thickness, which is sheared and thinned by even the smallest shear stress. Figures 4(c) and 4(d) demonstrate the effect of varying $B_{L}$, which controls the sharpness of the drop-off in the initial surfactant distribution function and therefore the size of the initial shear stress, and $x_{s}$, the peak location of the disturbance functions. The larger the initial shear stress or the longer the base flow is allowed to develop and steepen before encountering the disturbances, the larger the corresponding transient response.

Figures 5(a) and 5(b) depict a similar trend in $G_{\Psi}(t)$ as the parameters $\mathcal{C}$ and $\mathrm{Pe}_{s}$ are varied. The examples shown correspond to $K=10$, the mode exhibiting the largest overall amplitude in Fig. 4(a). The solutions clearly show that a decrease in $\mathcal{C}$ or an increase in $\mathrm{Pe}_{s}$, both of which produce a steeper advancing front, enhance the transient growth of disturbances. Other parameter choices which produce steeper fronts and correspondingly larger values of the surface curvature (and higher derivatives) behave similarly. ${ }^{20-22} \mathrm{In} \mathrm{ev-}$ ery case examined, provided $\mathcal{A}=0$, the transient growth eventually fades away and the system is asymptotically stable.

The physical mechanism leading to the large transient growth is linked to the extraction of energy from the spatially inhomogeneous waveform, as well as the evolution of a region of constant gradient in surfactant concentration behind the advancing front. ${ }^{20-22}$ The reader is referred to these references for an in depth discussion. This mechanism is quite different, however, from the fingering behavior observed at the leading edge of other free surface spreading problems, like the flow down an inclined plane $e^{30,31}$ or the thermocapillary driven spreading of a thin liquid film. ${ }^{32,33}$ In these other spreading processes, the instability occurs right at the leading edge and causes the spreading front to separate into long narrow rivulets which never undergo spreading, shielding, or tip-splitting. Furthermore, when the amplitude of the advancing ridge approaches values on the order of the thickness of the pre-existing liquid film, the fingering disappears altogether, in sharp contrast to a spreading surfactant film.

We have recently investigated the effect of van der Waals forces on the spreading profile for positive values of the Hamaker constant which promote film thinning. It is well known that the inclusion of such a force can lead to dewet- 

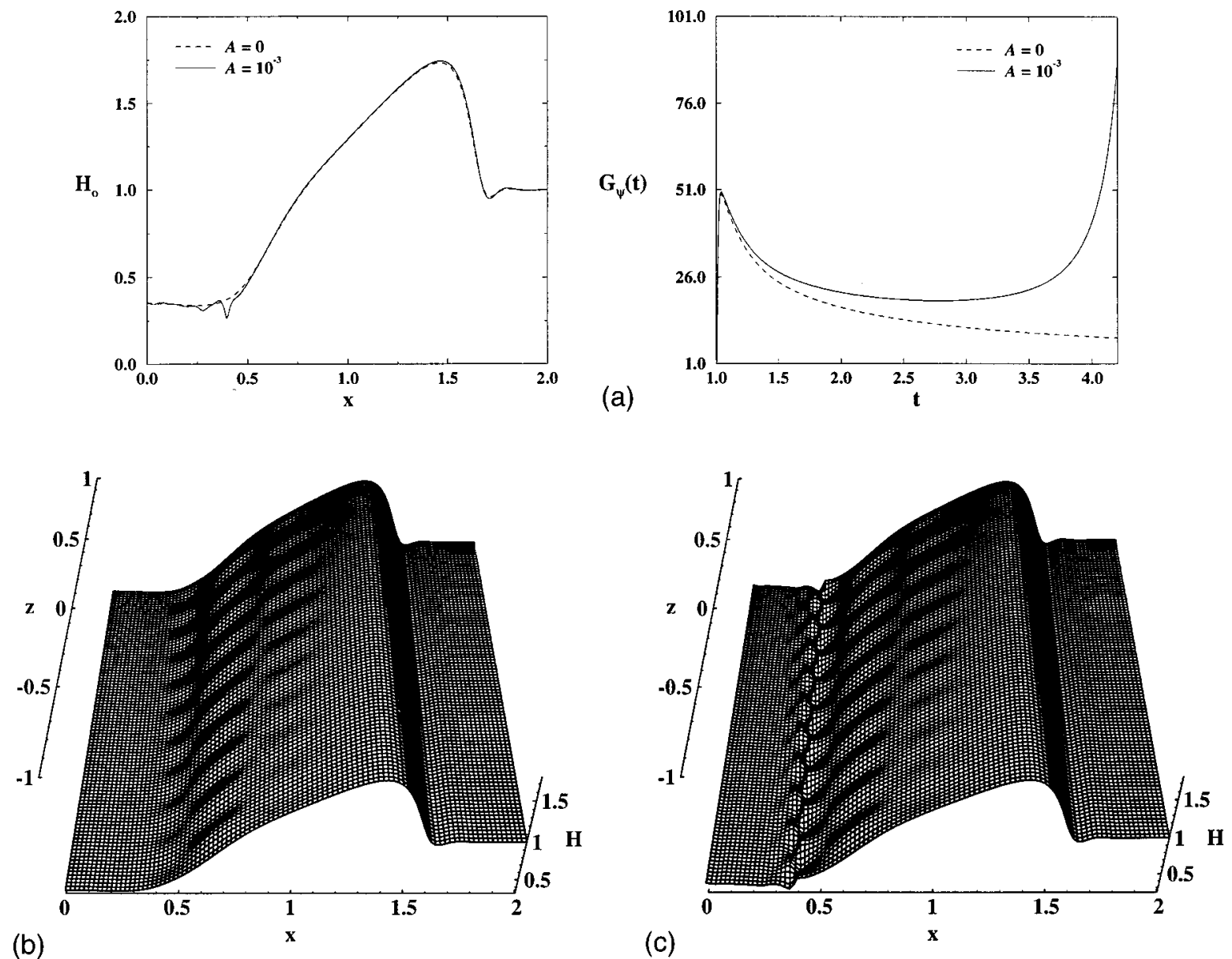

FIG. 6. The linearized response to disturbances in the presence of van der Waals thinning. (a) $H_{0}$ (at $t=4$ ) and $G_{\Psi}$ for $P_{s}=500, \mathcal{C}=10^{-5}, t_{0}=1, A_{L}=1$, $B_{L}=20, C_{L}=200, x_{0}=0.1, x_{s}=0.2$ and $N=301$. 3-D re-constructions of the total film thickness (for applied disturbance with $K=10$ ): (b) $\mathcal{A}=0$ and (c) $\mathcal{A}=10^{-3}$. The remaining parameter values are given in (a).

ting and film rupture. ${ }^{34-36} \mathrm{We}$ have found that the inclusion of this force in our calculations dramatically changes the behavior of $G_{\Psi}$ at intermediate times. As shown in Fig. 6 for parameter values $\mathcal{A}=10^{-3}, \mathrm{Pe}_{s}=500$, and $\mathcal{C}=10^{-5}$, once the film near the source undergoes sufficient thinning, van der Waals forces contribute to the amplification of disturbances, $G_{\Psi}(t)$, to produce a second even larger increase. (The parameter values for $A_{L}, B_{L}, C_{L}, x_{0}$, and $x_{s}$ are different than those used in Fig. 5 and were chosen simply to initiate more rapid film thinning near the source region.) This behavior suggests that other forces which promote film thinning will give rise to similar amplification in $G_{\Psi}(t)$. Unfortunately, the computations with nonzero $\mathcal{A}$ had to be stopped when the spatial gradients could no longer be resolved accurately. Different numerical techniques will be required to handle the onset of film rupture. Figures 6(b) and 6(c) depict the sum $H_{0}(x, t)+0.01 \Psi(x, t) \cos (K z)$ for a disturbance of $K=10$ at $\Delta t=3$ after deposition, i.e., $t=4$. Preliminary studies indicate that the streamwise and transverse components of the van der Waals term are destabilizing while capillary forces as well as streamwise Marangoni flow exert a stabilizing influence. ${ }^{20}$ The stabilizing effect of Marangoni stresses stems from their tendency to refill the surfactant expelled from the thinning region by van der Waals forces in agreement with previous studies. ${ }^{36}$ The addition of this type of disjoining pressure, then, gives rise to a spanwise film corrugation which localizes behind the advancing front, which persists to later times, and which resembles the patterns observed experimentally. More studies of this sort are required in order to determine whether the experimental patterns are evidence of a "momentary instability" which produces transient film corrugation driven by a coupling between Marangoni stresses and van der Waals forces. This coupling may eventually explain why the fingering patterns have never been observed in films thicker than a few millimeters, since the magnitude of the flux due to the van der Waals force is insignificant in thicker films. It may also explain why the fingering patterns become more ramified as the thickness of the underlying liquid film decreases.

\section{B. Nonlinear transient response}

For the parameter space investigated we have found that integration of the nonlinear equations produces film thickness and surfactant concentration profiles which appear almost identical to those obtained from the linearized theory shown in Fig. 3. In addition, the amplification ratios, $G_{\Psi}$ and $G_{\Phi}$, from the nonlinear computations shown in Fig. 7 can be directly compared to the ratios previously shown in Figs. 4(a) and 4(b). The overall shape and decay of the different 

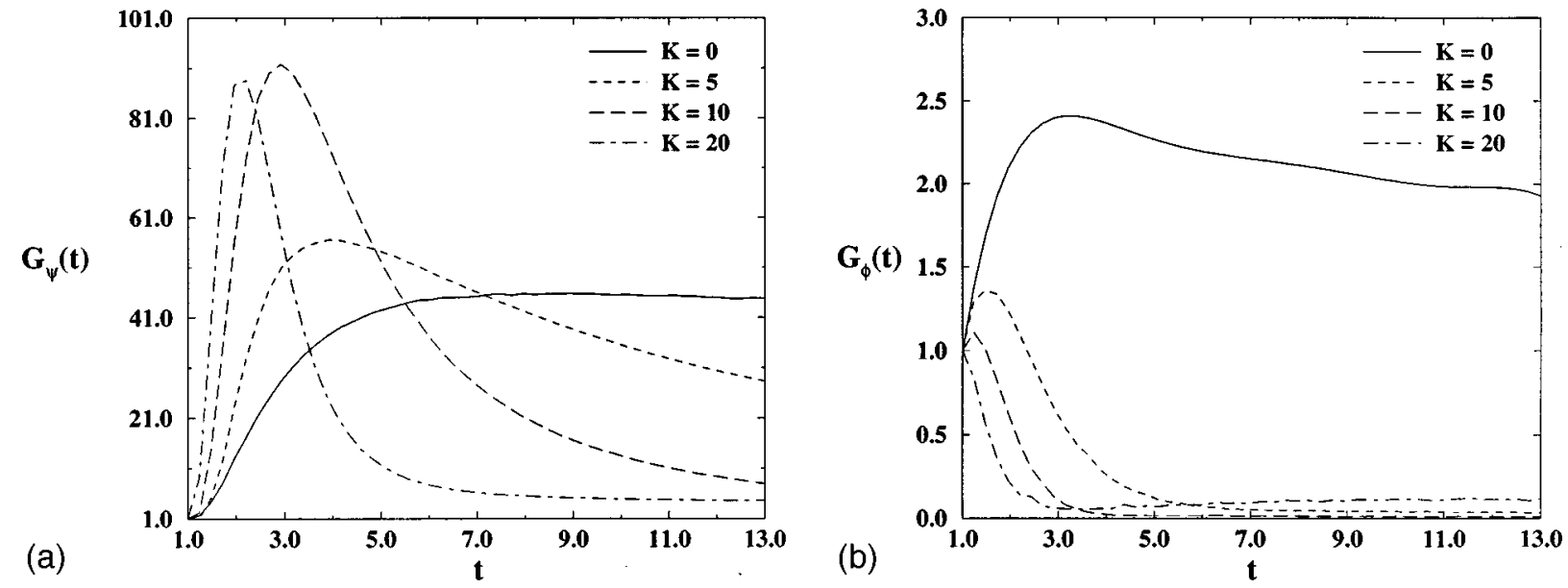

FIG. 7. Amplification ratios, $G_{\Psi}$ and $G_{\Phi}$, corresponding to the nonlinear transient growth, as a function of various wavenumber disturbances. Relevant parameter values: $\mathrm{Pe}_{s}=500, \mathcal{C}=10^{-5}, \mathcal{A}=0, \varepsilon=0.01, t_{0}=1, A_{L}=0.1, B_{L}=30, C_{N}=12, x_{0}=0.4, x_{s}=0.7, N_{x}=101$, and $N_{z}=201$.

wavenumber disturbances resemble each other very closely. The only observable differences are reflected in the maximum amplitude achieved. For example, the $K=20$ mode in the linear approximation achieves its maximum value of 81 in just under $t=2$, while in the nonlinear computations it reaches a value closer to 88 just after $t=2$. Surprisingly, even though the disturbances grow to be quite large and could potentially cause the nonlinear terms in the equations to contribute significantly, no such effect was observed. In addition, the nonlinear terms do not produce disturbances with wavenumbers different than the initial wavenumber. We have found that an applied disturbance with a given wavenumber will maintain that wavenumber throughout the spreading process, never evolving into a disturbance that contains subharmonics of the original frequency. This single frequency response was also observed by Tryggvason and Aref $^{37}$ and Tan and Homsy ${ }^{38}$ in their simulations of immiscible and miscible viscous fingering. Tan and Homsy suggested that this feature was caused by the transverse constraint of periodic boundary conditions which might pin the system to the original imposed wavelength and disallow the formation of subharmonics. The simulations of Tryggvason and Aref and Tan and Homsy did produce mode coupling when the original applied disturbance contained several fre-

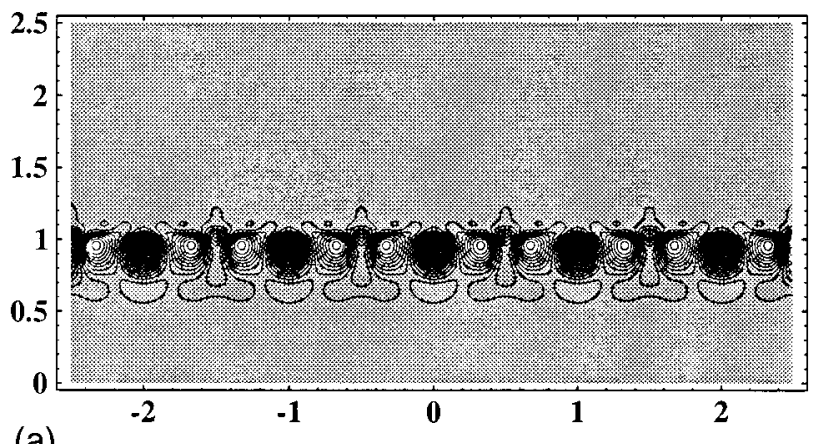

(a)
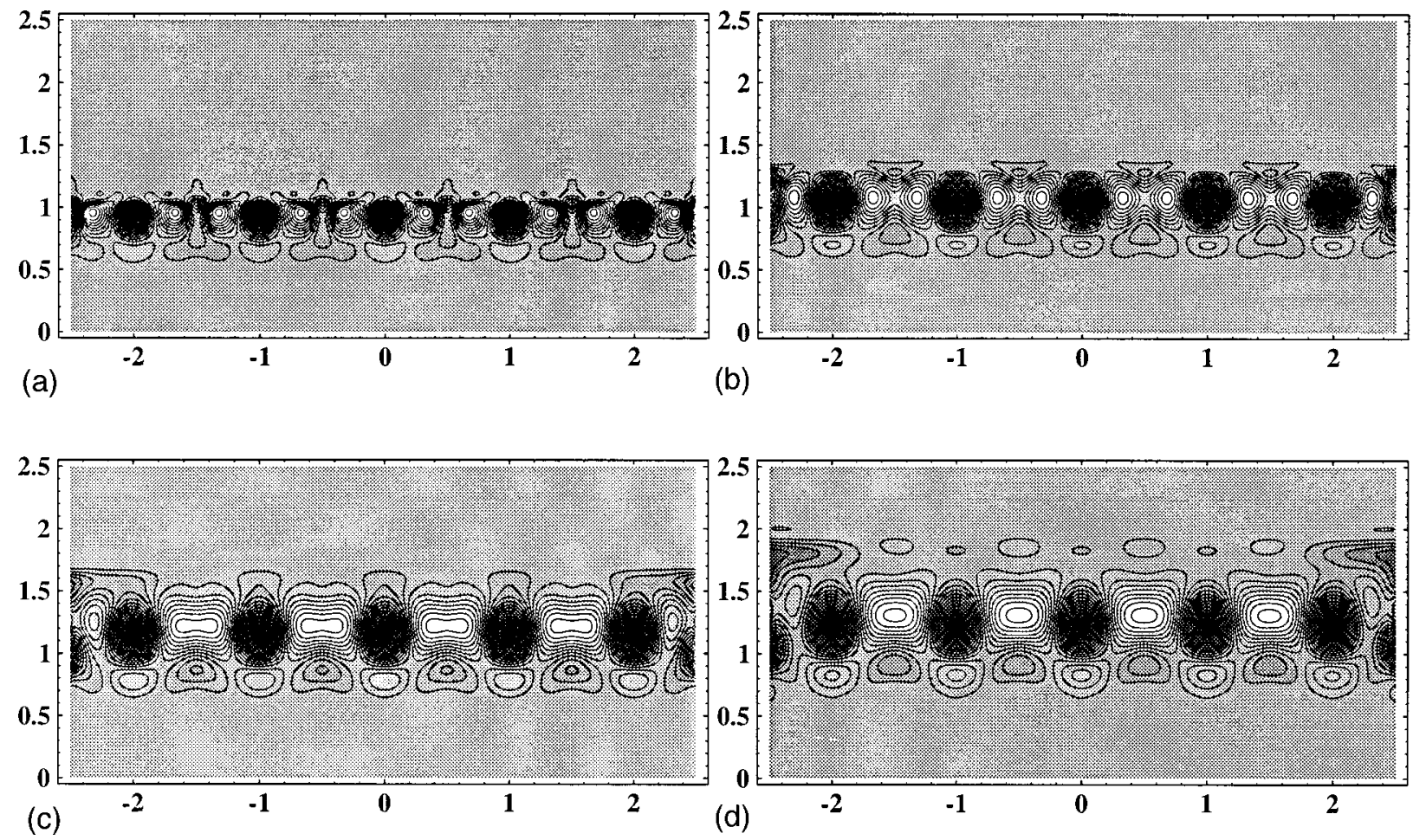

FIG. 8. Contour plots of the disturbance film thickness for a perturbation consisting of three wavenumbers, $K=5,10$, and 20 , applied with equal weight and propagated through the nonlinear equations. (a) $\Delta t=2$, (b) $\Delta t=4$, (c) $\Delta t=8$, and (d) $\Delta t=12$. The relevant parameter values are $\mathrm{Pe}_{s}=500, \mathcal{C}=10^{-5}, \mathcal{A}$ $=0, N_{x}=121, N_{z}=241$. The remaining parameter values are given in Fig. 7 . 


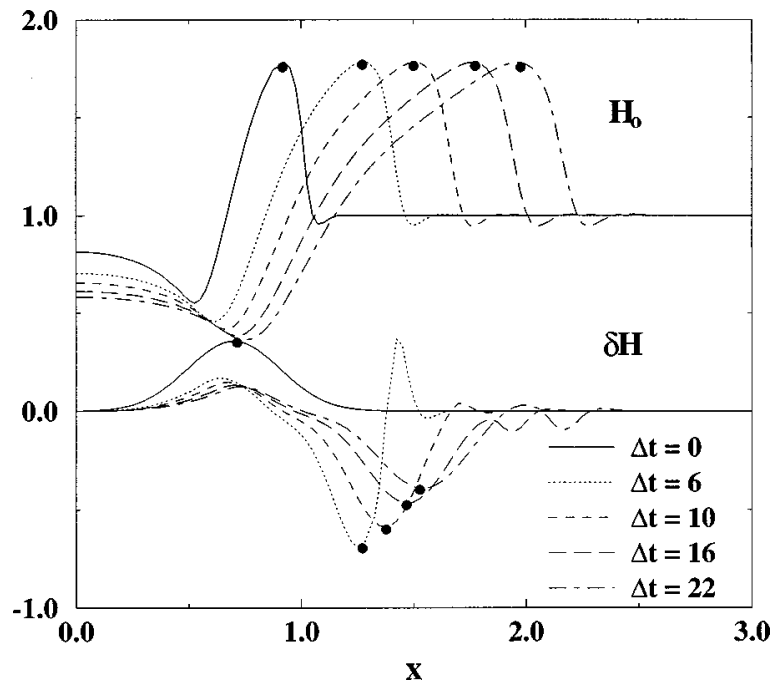

FIG. 9. A cross-sectional view of $H_{0}$ and $\delta H$ (for $K=5$ ) propagated through the nonlinear equations for a base state allowed first to evolve undisturbed through $t_{0}=3$. $\delta H$ has been magnified by a factor of 10 . Relevant parameter values: $\mathrm{Pe}_{s}=5000, \mathcal{C}=10^{-5}, \mathcal{A}=0$, and $\varepsilon=0.02$. The remaining parameter values are given in Fig. 7.

quencies, especially pairs or triplets of frequencies that could produce resonance. To investigate this possibility, we tested the response of our system of nonlinear equations to a disturbance consisting of three cosine modes [i.e., $m=3$ in $R(z)$ ] in Eq. (20) of equal but small amplitude consisting of the fundamental wavenumber $K=10$, its harmonic $K=20$, and its sub-harmonic $K=5$ for parameter values $\mathrm{Pe}_{s}=500$, $\mathcal{C}=10^{-5}$, and $\mathcal{A}=0$. Contour plots of the disturbance film thickness, obtained by subtraction of numerical solutions of Eqs. (1) and (2) with $\delta H>0$ and $\delta \Gamma>0$ from those with $\delta H=0$ and $\delta \Gamma=0$ (i.e., base state solutions), are shown in Fig. 8. White patches represent regions of highest elevation while black patches represent regions of lowest depression. While the disturbance film thickness initially reflects the presence of the three imposed wavenumbers, the system eventually sustains only the smallest imposed wavenumber, namely $K=5$. This tendency during spreading to move toward patterns with a smaller wavenumber was also observed in the linearized equations whose result is shown in Fig. 4. The smaller wavenumber corresponds to large wavelength disturbances and the trend toward wider "fingers" can be interpreted as a coalescence process. In the context of unstable viscous fingering, this trend has been called a modulatory instability and has been observed in both immiscible ${ }^{37}$ and miscible ${ }^{38}$ fingering.

Besides studying the behavior of the nonlinear equations to disturbances imposed at the very beginning of the spreading process, we also conducted studies in which the film thickness and surfactant concentration were allowed to evolve for some time before a disturbance was applied. In Fig. 9 is shown the evolution of the film thickness and its associated disturbance of $K=5$, which was placed on the film after it had evolved for a time $t_{0}=3$. The profiles represent cross-sectional views obtained for times $\Delta t=0,6,10$, 16 , and 22 after the disturbance was applied and for parameter values $\mathrm{Pe}_{s}=5000, \mathcal{C}=10^{-5}, \mathcal{A}=0$, and $\varepsilon=0.02$. The

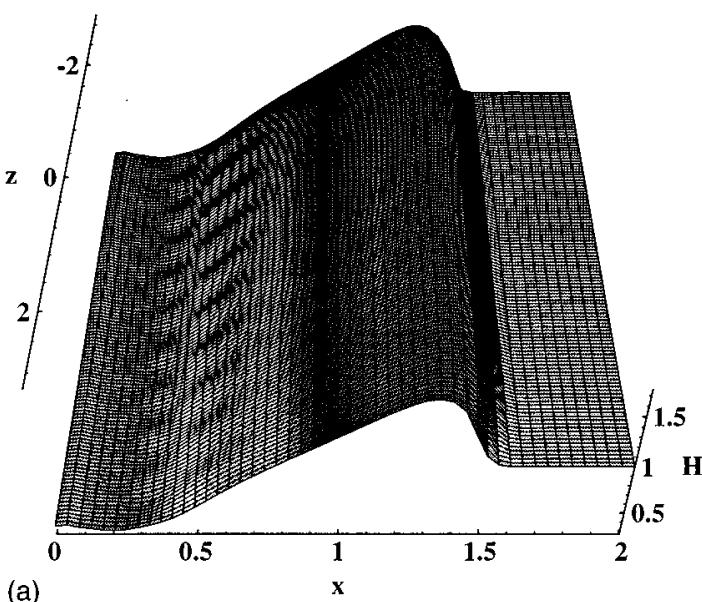

(a)

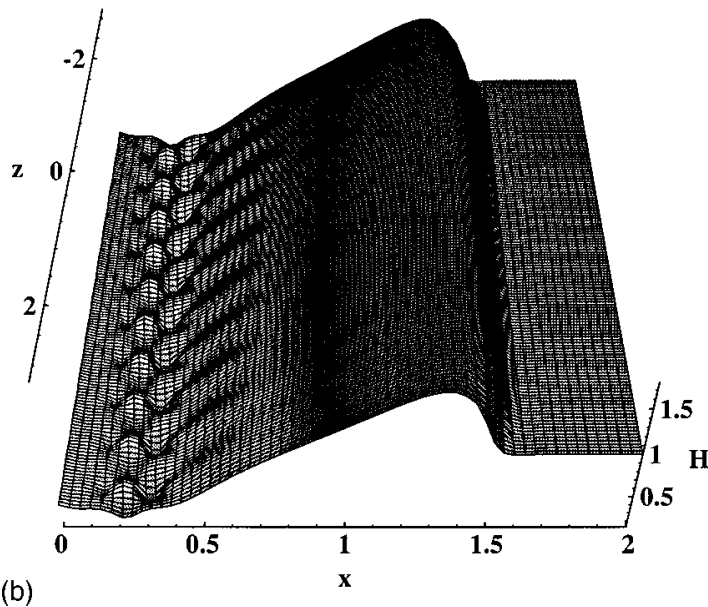

(b)

FIG. 10. 3-D images of the film thickness including an applied disturbance of $K=10$ propagated through the nonlinear equations in the presence of van der Waals forces. Relevant parameter values: $\mathrm{Pe}_{s}=500, \mathcal{C}=10^{-5}, t=4, t_{0}$ $=1, A_{L}=1, B_{L}=20, C_{N}=200, x_{0}=0.1, x_{s}=0.2, N_{x}=71$, and $N_{z}=141$. (a) $\mathcal{A}=0$ and (b) $\mathcal{A}=10^{-3}$.

magnitude of the disturbance film thickness has been exaggerated by a factor of ten to aid visualization. The black dots marking the peaks in $H_{0}$ and $\delta H$ are a guide to the eye for comparing the propagation speeds between the two waveforms. The initial disturbance in the film thickness is centered about the ramp-like portion but extends throughout the entire profile, affecting the region near the source all the way to the leading edge. As the wavefront in $H_{0}$ propagates forward, its shape broadens but the maximum value remains fairly constant for the period shown. The disturbance, $\delta H$, migrates toward the wavefront at early times but eventually cannot keep pace with the leading edge. After $\Delta t=6$, the peak in the disturbance has fallen behind the moving front continually slowing with respect to the leading edge in $H_{0}$. As seen previously in Fig. 3 for the linearized system and for different initial conditions, the disturbance localizes once again behind the moving front. Similarly, the inclusion of van der Waals forces in the nonlinear equations causes the disturbances to localize even further back toward the sharply thinned region near the source. The profiles in Fig. 10 are similar to those in Fig. 6 and reinforce the observation that 
the nonlinear terms do not contribute any new feature to the flow.

\section{CONCLUSION}

A previous study has shown that the linearized equations of motion governing the spontaneous spreading of a surfactant monolayer strip on a thin liquid film predict asymptotic stability. ${ }^{18,19}$ Recent work has suggested, however, that the transient response of this hydrodynamic system can be significant. ${ }^{21}$ We have shown that infinitesimal disturbances to the film thickness become amplified by several orders of magnitude, depending on the size of the initial shear stress, the spreading speed, and other physical parameters controlling the thickness of the advancing ridge and its degree of curvature (and higher order derivatives). In addition, the inclusion of a simplified van der Waals term in the linearized system of equations encourages strong thinning in the already thinnest parts of the spreading film intensifying the transient growth at intermediate times. The relative energy of the disturbances produced in the absence of van der Waals forces is always observed to undergo large transient growth and eventual decay. When the computations are performed with the van der Waals term, the disturbances also grow and decay but at intermediate times suddenly experience a second boost in energy. This secondary amplification reflects a type of resonant behavior between the thinning caused by the spreading film (due to Marangoni stresses) and the van der Waals driven thinning which enhances transverse corrugations established previously by the rapid Marangoni flow. Unfortunately, the transient computations were terminated once the spatial gradients and higher derivatives of the free interface shape could no longer be resolved accurately. We are investigating the use of other numerical techniques better able to handle this problem.

We have also investigated the stability of the nonlinear system of equations in the film thickness and surfactant concentration by direct numerical simulations. For the parameter set used, we could discern no major influence from the nonlinear terms ignored in the linearized description. The undisturbed and disturbed profiles look remarkably similar, as does the system's transient behavior as a whole. We have not excluded the possibility that larger amplitude disturbances than those applied here could affect the system in ways not evident in the linearized equations. The nonlinear equations were subjected to disturbances of either one wavelength or multiple wavelengths in order to probe the existence of mode coupling or splitting. Application of a single wavelength disturbance never produced other harmonics during the spreading process. The application of multiple wavelengths produced surface profiles that in time tended to select out the largest wavelength originally present. This feature could also be inferred from the linearized description at intermediate and late times which predicted that the mode with the largest amplification ratio always tended toward the mode with the largest wavelength. This tendency may be describing a coalescence process in which digitated patterns of various wavelengths coalesce to produce wider "fingers." Finally, the inclusion of van der Waals thinning into the nonlinear equa- tions of motion produced digitated surface structures which resemble closely the patterns obtained with the linearized equations. It appears that the effect induced by including this term in the linearized set of equations is much more dramatic than any effects produced by considering the fully nonlinear set of equations.

Our parameter range for the nonlinear studies indicate that the nonlinear description offers little physical insight not already present in the linearized description. The most powerful effect on the surfactant coated film appears to lie with the coupling between the already thinned film produced by the rapid Marangoni spreading and the even stronger thinning later induced by the van der Waals forces. It is as if the film has been primed by the first process to produce conditions which intensify disturbances that would normally decay. In addition, the rapid thinning strongly localizes disturbances in the thinnest parts of the film. A review of the experimental literature reveals that the digitated patterns most easily appear on very thin films. For example, the branched patterns shown in Fig. 1 occur in films whose thickness makes them susceptible to van der Waals forces.

We hope our studies will focus attention on the significance of van der Waals forces on the spreading process and encourage experimentation which can ascertain the validity of this claim. In addition, we have initiated numerical work to study the effect of white noise on the spreading dynamics and to investigate parameter values which can lead to branching and tip-splitting, processes more closely related to nonlinear phenomena in hydrodynamic systems.

\section{ACKNOWLEDGMENT}

This work was supported by a National Science Foundation CAREER award through Grant No. CTS-9624776.

${ }^{1}$ V. G. Levich, Physicochemical Hydrodynamics (Prentice-Hall, Englewood Cliffs, NJ, 1962).

${ }^{2}$ A. Marmur and M. D. Lelah, "The spreading of aqueous surfactant solutions on glass,', Chem. Eng. Commun. 13, 133 (1981).

${ }^{3}$ S. M. Troian, X. L. Wu, and S. A. Safran, "Fingering instability in thin wetting films,', Phys. Rev. Lett. 62, 1496 (1989).

${ }^{4}$ B. Frank and S. Garoff, "Origins of the complex motion of advancing surfactant solutions,', Langmuir 11, 87 (1995).

${ }^{5} \mathrm{~S}$. He and J. Ketterson, "Surfactant driven spreading of a liquid on a vertical surface,', Phys. Fluids A 7, 2640 (1995).

${ }^{6} \mathrm{~S}$. Bardon et al., "Structure and dynamics of liquid films on solid surfaces," Faraday Discuss. 104, 307 (1996).

${ }^{7}$ S. M. Troian, X. L. Wu, and S. A. Safran, in Phase Transitions in Soft Condensed Matter, NATO ASI Series Vol. 211, edited by T. Riste and D. Sherrington (Plenum, New York, 1989).

${ }^{8}$ P. G. Saffman and G. I. Taylor, "The penetration of a fluid into a porous medium or Hele-Shaw cell containing a more viscous liquid,' Proc. R. Soc. London 245, 312 (1958).

${ }^{9}$ G. M. Homsy, "Viscous fingering in porous media,' Annu. Rev. Fluid Mech. 19, 271 (1987).

${ }^{10}$ A. M. Pereira, B.S.E. thesis, Princeton University, Princeton, NJ, 1995.

${ }^{11}$ S. M. Troian, E. Herbohlzheimer, and S. A. Safran, "Model for the fingering instability of spreading surfactant drops,' Phys. Rev. Lett. 65, 333 (1990).

${ }^{12}$ M. E. Borgas and J. B. Grotberg, "Monolayer flow on a thin film," J. Fluid Mech. 193, 151 (1988).

${ }^{13}$ D. P. Gaver and J. B. Grotberg, "The dynamics of a localized surfactant on a thin film,' J. Fluid Mech. 213, 127 (1990).

${ }^{14}$ O. E. Jensen and J. B. Grotberg, "Insoluble surfactant spreading on a thin viscous thin: Shock evolution and rupture,' J. Fluid Mech. 240, 259 (1992). 
${ }^{15}$ F. F. Espinosa, A. H. Shapiro, J. J. Fredberg, and R. D. Kamm, "Spreading of exogenous surfactant in an airway,' J. Appl. Physiol. 75, 2028 (1993).

${ }^{16}$ J. B. Grotberg, D. Halpern, and O. E. Jensen, "Interaction of exogenous and endogenous surfactant: spreading-rate effects,' J. Appl. Physiol. 78, 750 (1995).

${ }^{17}$ O. E. Jensen, D. Halpern, and J. B. Grotberg, in Surface-Tension-Driven Flows, Proceedings of the ASME, Applied Mechanics Division, edited by G. P. Neitzel and E. M. K. Smith (The Applied Mechanics Division, ASME, New York, 1993), Vol. 170, p. 47.

${ }^{18}$ O. K. Matar and S. M. Troian, in Dynamics in Small Confining Systems III, Materials Research Society Symposium Proceedings, edited by J. M. Drake, J. Klafter, and E. R. Kopelman (Materials Research Society, Boston, 1996), Vol. 464, p. 237.

${ }^{19}$ O. K. Matar and S. M. Troian, "Linear instability analysis of an insoluble surfactant monolayer spreading on a thin liquid film,' Phys. Fluids A 9, 3645 (1997).

${ }^{20}$ O. K. Matar, Ph.D. thesis, Princeton University, Princeton, NJ, 1998.

${ }^{21}$ O. K. Matar and S. M. Troian, "Growth of non-modal transient structures during the spreading of surfactant coated films,'” Phys. Fluids A 10, 1234 (1998).

${ }^{22}$ O. K. Matar and S. M. Troian, "Transient response of a surfactant monolayer spreading on a thin liquid film: Mechanism for amplification of disturbances,', submitted to Phys. Fluids.

${ }^{23}$ L. N. Trefethen, A. E. Trefethen, S. C. Reddy, and T. A. Driscoll, "Hydrodynamic stability without eigenvalues,' Science 261, 578 (1993).

${ }^{24}$ B. F. Farrell and P. J. Ioannou, "Generalized stability theory. Part II: Nonautonomous operators,'” J. Atmos. Sci. 53, 2041 (1996).

${ }^{25} \mathrm{~W}$. M. Orr, "The stability or instability of the steady motions of a perfect liquid and of a viscous liquid. Part II: A viscous liquid,' Proc. R. Ir. Acad. Sect. A, Math. Astron. Phys. Sci. 27, 69 (1907).
${ }^{26}$ D. R. Wilson and A. F. Jones, "The entry of a falling film into a pool and the air-entrainment problem,'” J. Fluid Mech. 128, 219 (1983).

${ }^{27}$ S. F. Shen, "Some considerations of the laminar stability of incompressible time-dependent basic flows," J. Aerosp. Sci. 28, 397 (1961).

${ }^{28}$ W. E. Schiesser, The Numerical Method of Lines (Academic, San Diego, 1991).

${ }^{29}$ A. C. Hindmarsh, in Scientific Computing, edited by R. S. Stepleman (North-Holland, Amsterdam, 1983), p. 55.

${ }^{30}$ S. M. Troian, E. Herbohlzheimer, S. A. Safran, and J. F. Joanny, "Fingering instabilities of driven spreading films," Europhys. Lett. 10, 25 (1989).

${ }^{31}$ A. L. Bertozzi and M. P. Brenner, "Linear stability analysis and transient growth in driven contact lines," Phys. Fluids A 9, 530 (1997)

${ }^{32}$ D. E. Kataoka and S. M. Troian, "A theoretical study of instabilities at the advancing front of thermally driven coating films," J. Colloid Interface Sci. 192, 350 (1997).

${ }^{33}$ D. E. Kataoka and S. M. Troian, "Stabilizing the advancing front of thermally driven coating films,' J. Colloid Interface Sci. 203, 335 (1998).

${ }^{34}$ E. Ruckenstein and R. K. Jain, "Spontaneous rupture of thin liquid films," J. Chem. Soc., Faraday Trans. 70, 132 (1974).

${ }^{35}$ M. B. Williams and S. H. Davis, "Nonlinear theory of film rupture,' J. Colloid Interface Sci. 90, 220 (1982)

${ }^{36}$ C.-C. Hwang et al., "Nonlinear rupture theory of a thin liquid film with insoluble surfactant,', J. Fluids Eng. 120, 598 (1998).

${ }^{37} \mathrm{G}$. Tryggvason and H. Aref, "'Finger-interaction mechanisms in stratified Hele-Shaw flow,', J. Fluid Mech. 154, 287 (1985).

${ }^{38}$ C. T. Tan and G. M. Homsy, "'Simulation of nonlinear viscous fingering in miscible displacement,', Phys. Fluids A 31, 1330 (1988). 